\title{
Three-Dimensional 1-Bend Graph Drawings
}

\author{
Pat Morin \\ School of Computer Science \\ Carleton University \\ Ottawa, Canada \\ morin@scs.carleton.ca \\ David R. Wood \\ Departament de Matemàtica Aplicada II \\ Universitat Politècnica de Catalunya \\ Barcelona, Spain \\ david.wood@upc.edu
}

\begin{abstract}
We consider three-dimensional grid-drawings of graphs with at most one bend per edge. Under the additional requirement that the vertices be collinear, we prove that the minimum volume of such a drawing is $\Theta(c n)$, where $n$ is the number of vertices and $c$ is the cutwidth of the graph. We then prove that every graph has a three-dimensional grid-drawing with $\mathcal{O}\left(n^{3} / \log ^{2} n\right)$ volume and one bend per edge. The best previous bound was $\mathcal{O}\left(n^{3}\right)$.
\end{abstract}

\begin{tabular}{|llll|}
\hline $\begin{array}{l}\text { Article Type } \\
\text { concise paper }\end{array}$ & Communicated by & Submitted & Revised \\
\hline
\end{tabular}

Presented at the 16th Canadian Conference on Computational Geometry (CCCG '04), Concordia University, Montréal, Canada, August 9-11, 2004.

Research of Pat Morin supported by NSERC.

Research of David Wood supported by the Government of Spain grant MEC SB20030270, and partially completed at Carleton University, Canada. 


\section{Introduction}

We consider undirected, finite, and simple graphs $G$ with vertex set $V(G)$ and edge set $E(G)$. The number of vertices and edges of $G$ are respectively denoted by $n=|V(G)|$ and $m=|E(G)|$.

Graph drawing is concerned with the automatic generation of aesthetically pleasing geometric representations of graphs. Graph drawing in the plane is well-studied (see [5, 17]). Motivated by experimental evidence suggesting that displaying a graph in three dimensions is better than in two [20,21], and applications including information visualisation [20], VLSI circuit design [18], and software engineering [22], there is a growing body of research in three-dimensional graph drawing.

A three-dimensional polyline grid-drawing of a graph, henceforth called a polyline drawing, represents the vertices by distinct points in $\mathbb{Z}^{3}$ (called gridpoints), and represents each edge as a polyline between its endpoints with bends (if any) also at gridpoints, such that distinct edges only intersect at common endpoints, and each edge only intersects a vertex that is an endpoint of that edge. A polyline drawing with at most $b$ bends per edge is called a $b$-bend drawing. A 0-bend drawing is called a straight-line drawing.

A folklore result states that every graph has a straight-line drawing. Thus we are interested in optimising certain measures of the aesthetic quality of such drawings. The bounding box of a polyline drawing is the minimum axis-aligned box containing the drawing. If the bounding box has side lengths $X-1, Y-1$ and $Z-1$, then we speak of an $X \times Y \times Z$ polyline drawing with volume $X \cdot Y \cdot Z$. That is, the volume of a polyline drawing is the number of gridpoints in the bounding box. This definition is formulated so that two-dimensional drawings have positive volume. This paper continues the study of upper bounds on the volume and number of bends per edge in polyline drawings. The volume of straight-line drawings has been widely studied $[1-3,6,9,11,12,14,16,19]$. Only recently have (non-orthogonal) polyline drawings been considered [4, 12, 13]. Table 1 summarises the best known upper bounds on the volume and bends per edge in polyline drawings.

Cohen et al.[3] proved that the complete graph $K_{n}$ (and hence every $n$ vertex graph) has a straight-line drawing with $\mathcal{O}\left(n^{3}\right)$ volume, and that $\Omega\left(n^{3}\right)$ volume is necessary. Dyck et al.[13] recently proved that $K_{n}$ has a 2-bend drawing with $\mathcal{O}\left(n^{2}\right)$ volume. The same conclusion can be reached from the $\mathcal{O}(q n)$ volume bound of Dujmović and Wood[12], since trivially every graph has a $(n-1)$-queue layout. Dyck et al.[13] asked the interesting question: what is the minimum volume in a 1-bend drawing of $K_{n}$ ? The best known upper bound at the time was $\mathcal{O}\left(n^{3}\right)$, while $\Omega\left(n^{2}\right)$ is the best known lower bound. (Bose et al.[1] proved that all polyline drawings have $\Omega(n+m)$ volume.)

In this paper we prove two results. The first concerns collinear polyline drawings in which all the vertices are in a single line. Let $G$ be a graph, and let $\sigma$ be a linear order of $V(G)$. Let $L_{\sigma}(e)$ and $R_{\sigma}(e)$ denote the endpoints of each edge $e$ such that $L_{\sigma}(e)<_{\sigma} R_{\sigma}(e)$. For each vertex $v \in V(G)$, the set $\left\{e \in E(G): L_{\sigma}(e) \leq_{\sigma} v<_{\sigma} R_{\sigma}(e)\right\}$ is called the cut in $\sigma$ at $v$. The cutwidth of $\sigma$ 
Table 1: Volume of 3D polyline drawings of $n$-vertex graphs with $m \geq n$ edges.

\begin{tabular}{lrll}
\hline graph family & bends per edge & volume & reference \\
\hline arbitrary & 0 & $\mathcal{O}\left(n^{3}\right)$ & Cohen et al.[3] \\
arbitrary & 0 & $\mathcal{O}\left(m^{4 / 3} n\right)$ & Dujmović and Wood[11] \\
maximum degree $\Delta$ & 0 & $\mathcal{O}(\Delta m n)$ & Dujmović and Wood[11] \\
bounded chromatic number & 0 & $\mathcal{O}\left(n^{2}\right)$ & Pach et al.[19] \\
bounded chromatic number & 0 & $\mathcal{O}\left(m^{2 / 3} n\right)$ & Dujmović and Wood[11] \\
bounded maximum degree & 0 & $\mathcal{O}\left(n^{3 / 2}\right)$ & Dujmović and Wood[11] \\
$H$-minor free $(H$ fixed) & 0 & $\mathcal{O}\left(n^{3 / 2}\right)$ & Dujmović and Wood[11] \\
bounded treewidth & 0 & $\mathcal{O}(n)$ & Dujmović et al.[9] \\
$k$-colourable $q$-queue & 1 & $\mathcal{O}(k q m)$ & Dujmović and Wood[12] \\
arbitrary & 1 & $\mathcal{O}(n m)$ & Dujmović and Wood[12] \\
cutwidth $c$ & 1 & $\mathcal{O}(c n)$ & Theorem 1 \\
arbitrary & 1 & $\mathcal{O}\left(n^{3} / \log ^{2} n\right)$ & Theorem 2 \\
$q$-queue & 2 & $\mathcal{O}(q n)$ & Dujmović and Wood[12] \\
$q$-queue (constant $\epsilon>0)$ & $\mathcal{O}(1)$ & $\mathcal{O}\left(m q^{\epsilon}\right)$ & Dujmović and Wood[12] \\
$q$-queue & $\mathcal{O}(\log q)$ & $\mathcal{O}(m \log q)$ & Dujmović and Wood[12] \\
\hline
\end{tabular}

is the maximum size of a cut in $\sigma$. The cutwidth of $G$ is the minimum cutwidth of a linear order of $V(G)$. Cutwidth is a widely studied graph parameter (see $[7])$.

Theorem 1. Let $G$ be a graph with $n$ vertices and cutwidth $c$. The minimum volume for a 1-bend collinear drawing of $G$ is $\Theta(c n)$.

Theorem 1 represents a qualitative improvement over the $\mathcal{O}(n m)$ volume bound for 1-bend drawings by Dujmović and Wood[12]. Our second result improves the best known upper bound for 1-bend drawings of $K_{n}$.

Theorem 2. Every complete graph $K_{n}$, and hence every n-vertex graph, has a 1-bend $\mathcal{O}(\log n) \times \mathcal{O}(n) \times \mathcal{O}\left(n^{2} / \log ^{3} n\right)$ drawing with $\mathcal{O}\left(n^{3} / \log ^{2} n\right)$ volume.

It is not straightforward to compare the volume bound in Theorem 2 with the $\mathcal{O}(k q m)$ bound by Dujmović and Wood[12] for $k$-colourable $q$-queue graphs (see Table 1). However, since $k \leq 4 q$ and $m \leq 2 q n$ (see [10]), we have that $\mathcal{O}(k q m) \subseteq$ $\mathcal{O}\left(q^{3} n\right)$, and thus the $\mathcal{O}(k q m)$ bound by Dujmović and Wood[12] is no more than the bound in Theorem 2 whenever the graph has a $\mathcal{O}\left((n / \log n)^{2 / 3}\right)$-queue layout. On the other hand, $k q m \geq m^{2} / n$. So for dense graphs with $\Omega\left(n^{2}\right)$ edges the $\mathcal{O}(k q m)$ bound by Dujmović and Wood[12] is cubic (in $n$ ), and the bound in Theorem 2 is necessarily smaller. In particular, Theorem 2 provides a partial solution to the above-mentioned open problem of Dyck et al.[13] regarding the minimum volume of a 1-bend drawing of $K_{n}$. 


\section{Proof of Theorem 1}

First we prove the lower bound in Theorem 1.

Lemma 1. Let $G$ be a graph with $n$ vertices and cutwidth $c$. Then every 1-bend collinear drawing of $G$ has at least cn/2 volume.

Proof. Consider a 1-bend collinear drawing of $G$ in an $X \times Y \times Z$ bounding box. Let $L$ be the line containing the vertices. If $L$ is not contained in a grid-plane, then $X, Y, Z \geq n$, and the volume is at least $n^{3} \geq c n$.

Now assume, without loss of generality, that $L$ is contained in the $Z=0$ plane. Let $\sigma$ be a linear order of the vertices determined by $L$. Let $B$ be the set of bends corresponding to the edges in the largest cut in $\sigma$. Then $|B| \geq c$. For every line $L^{\prime}$ parallel to $L$, there is at most one bend in $B$ on $L^{\prime}$, as otherwise there is a crossing.

First suppose that $L$ is axis-parallel. Without loss of generality, $L$ is the $X$-axis. Then $X \geq n$. The gridpoints in the bounding box can be covered by $Y Z$ lines parallel to $L$. Thus $Y Z \geq|B| \geq c$, and the volume $X Y Z \geq c n$.

Now suppose that $L$ is not axis-parallel. Thus $X \geq n$ and $Y \geq n$. The gridpoints in the bounding box can be covered by $Z(X+Y)$ lines parallel to $L$. Thus $Z(X+Y) \geq|B| \geq c$, and the volume $X Y Z \geq X Y c /(X+Y) \geq c n / 2$.

To prove the upper bound in Theorem 1 we will need the following lemma, which is a slight generalisation of a well known result. (For example, Pach et al.[19] proved the case $X=Y$ ). We say two gridpoints $v$ and $w$ in the plane are visible if the segment $v w$ contains no other gridpoint.

Lemma 2. The number of gridpoints $\{(x, y): 1 \leq x \leq X, 1 \leq y \leq Y\}$ that are visible from the origin is at least $3 X Y / 2 \pi^{2}$.

Proof. Without loss of generality $X \leq Y$. Let $N$ be the desired number of gridpoints. For each $1 \leq x \leq X$, let $N_{x}$ be the number of gridpoints $(x, y)$ that are visible from the origin, such that $1 \leq y \leq Y$. A gridpoint $(x, y)$ is visible from the origin if and only if $x$ and $y$ are coprime. Let $\phi(x)$ be the number of positive integers less than $x$ that are coprime with $x$ (Euler's $\phi$ function). Thus $N_{x} \geq \phi(x)$, and

$$
N=\sum_{x=1}^{X} N_{x} \geq \sum_{x=1}^{X} \phi(x) \approx \frac{3 X^{2}}{\pi^{2}} .
$$

(See [15] for a proof that $\sum_{x=1}^{X} \phi(x) \approx 3 X^{2} / \pi^{2}$.) If $X \geq Y / 2$, then $N \geq$ $3 X Y / 2 \pi^{2}$, and we are done. Now assume that $Y \geq 2 X$. If $x$ and $y$ are coprime, then $x$ and $y+x$ are coprime. Thus $N_{x} \geq\lfloor Y / x\rfloor \cdot \phi(x)$. Thus,

$$
N \geq \sum_{x=1}^{X}\left\lfloor\frac{Y}{x}\right\rfloor \cdot \phi(x) \geq\left(\frac{Y-X}{X}\right) \sum_{x=1}^{X} \phi(x) \approx \frac{3(Y-X) X}{\pi^{2}} \geq \frac{3 X Y}{2 \pi^{2}}
$$


Now we prove the following strengthening of the upper bound in Theorem 1.

Lemma 3. Let $G$ be a graph with $n$ vertices and cutwidth $c$. For all integers $X \geq 1, G$ has a 1-bend collinear $X \times \mathcal{O}(c / X) \times n$ drawing with the vertices on the $Z$-axis. The volume is $\mathcal{O}(\mathrm{cn})$.

Proof. Let $\sigma$ be a vertex ordering of $G$ with cutwidth $c$. For all pairs of distinct edges $e$ and $f$, say $e \prec f$ whenever $R_{\sigma}(e) \leq_{\sigma} L_{\sigma}(f)$. Then $\preceq$ is a partial order on $E(G)$. A chain (respectively, antichain) in a partial order is a set of pairwise comparable (incomparable) elements. Thus an antichain in $\preceq$ is exactly a cut in $\sigma$. Dilworth's Theorem [8] states that every partial order with no $(k+1)$ element antichain can be partitioned into $k$ chains. Thus there is a partition of $E(G)$ into chains $E_{1}, E_{2}, \ldots, E_{c}$, such that each $E_{i}=\left(e_{i, 1}, e_{i, 2}, \ldots, e_{i, k_{i}}\right)$ and $R_{\sigma}\left(e_{i, j}\right) \leq_{\sigma} L_{\sigma}\left(e_{i, j+1}\right)$ for all $1 \leq j \leq k_{i}-1$.

By Lemma 2 with $Y=\left\lceil 4 \pi^{2} c / 3 X\right\rceil$, there is a set $S=\left\{\left(x_{i}, y_{i}\right): 1 \leq i \leq\right.$ $\left.c, 1 \leq x_{i} \leq X, 1 \leq y_{i} \leq Y\right\}$ of gridpoints that are visible from the origin. Position the $i$ th vertex in $\sigma$ at $(0,0, i)$ on the $Z$-axis, and position the bend for each edge $e_{i, j}$ at $\left(x_{i}, y_{i}, j\right)$. Edges in distinct chains are contained in distinct planes that only intersect in the $Z$-axis. Thus such edges do not cross. Edges within each chain $E_{i}$ do not cross since no two edges in $E_{i}$ are nested or crossing in $\sigma$, and the $Z$-coordinates of the bends of the edges in $E_{i}$ agrees with the order of their endpoints on the $Z$-axis, as (imprecisely) illustrated in Figure 1 . The bounding box is $X \times\left\lceil 4 \pi^{2} c / 3 X\right\rceil \times n$, since each chain has at most $n-1$ edges.

The constants in Lemma 3 can be tweaked as follows.

Lemma 4. Let $G$ be a graph with $n$ vertices and cutwidth $c$. Then $G$ has a 1 -bend collinear $3 \times\lceil(c-2) / 2\rceil \times n$ drawing. The volume is at most $3(c-1) n / 2$.

Proof. Let $S=\{(-1,0),(1,0)\} \cup\{(x, y): y \in\{-1,1\},-1 \leq x \leq\lceil(c-6) / 2\rceil\}$. Then $S$ consists of at least $c$ gridpoints that are visible from the origin. The result follows from the proof of Lemma 3.

Since the cutwidth of $K_{n}$ is $n^{2} / 4$ we have:

Corollary 1. The minimum volume for a 1-bend collinear drawing of the complete graph $K_{n}$ is $\Theta\left(n^{3}\right)$. For all $X \geq 1, K_{n}$ has a 1-bend collinear $X \times$ $\mathcal{O}\left(n^{2} / X\right) \times n$ drawing with the vertices on the $Z$-axis. Furthermore, $K_{n}$ has a 1-bend collinear $3 \times\left\lceil n^{2} / 8\right\rceil \times n$ drawing with volume at most $3 n^{3} / 8$.

\section{Proof of Theorem 2}

Let $P=\left\lceil\frac{1}{2} \log _{4} n\right\rceil$ and $Q=\lceil n / P\rceil$. Let $V\left(K_{n}\right)=\left\{v_{a, i}: 1 \leq a \leq P, 1 \leq i \leq Q\right\}$. Position each vertex $v_{a, i}$ at

$$
(2 a, a Q+i, 0)
$$




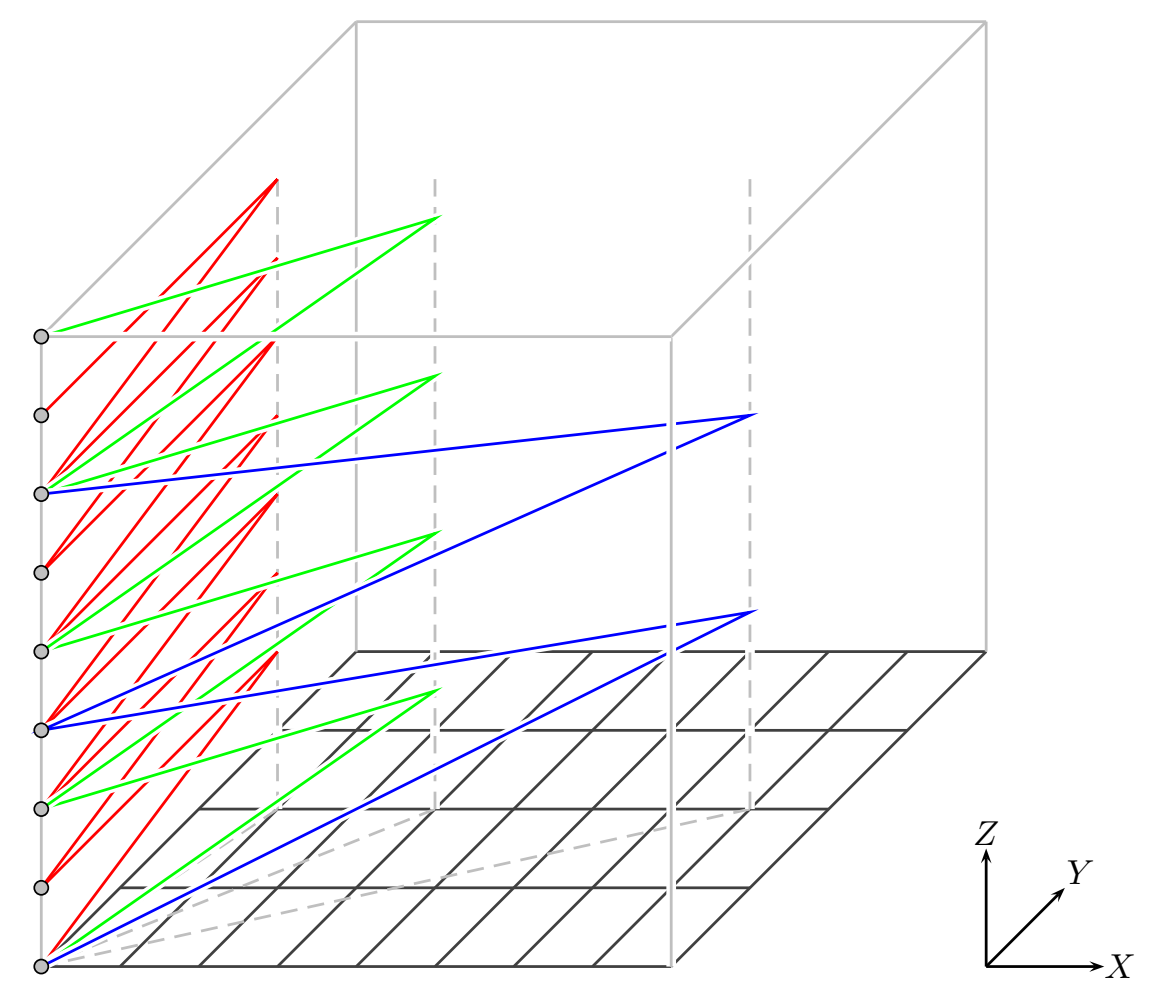

Figure 1: Construction of collinear 1-bend drawing in Lemma 3.

For each $1 \leq a \leq P$, the set of vertices $\left\{v_{a, i}: 1 \leq i \leq Q\right\}$ induces a complete graph $K_{Q}$, which is drawn using Corollary 1 (with the dimensions permuted) in the box

$$
[2 a, 2 a+P] \times[a Q+1,(a+1) Q] \times\left[0,-c Q^{2} / P\right],
$$

for some constant $c$. For all $1 \leq a<b \leq P$, orient each edge $e=\left(v_{a, i}, v_{b, j}\right)$, and position the bend for $e$ at

$$
r_{e}=\left(2 a+1, b Q+j, 4^{P-a} Q-i\right),
$$

as (imprecisely) illustrated in Figure 2. We say $v_{a, i} r_{e}$ is an outgoing segment at $v_{a, i}$, and $r_{e} v_{b, j}$ is an incoming segment at $v_{b, j}$.

Thus the bounding box is $\mathcal{O}(P) \times \mathcal{O}(n) \times \mathcal{O}\left(4^{P} Q+Q^{2} / P\right)$, which is $\mathcal{O}(\log n) \times$ $\mathcal{O}(n) \times \mathcal{O}\left(n^{3 / 2} / \log n+n^{2} / \log ^{3} n\right)$, which is $\mathcal{O}(\log n) \times \mathcal{O}(n) \times \mathcal{O}\left(n^{2} / \log ^{3} n\right)$. Hence the volume is $\mathcal{O}\left(n^{3} / \log ^{2} n\right)$. It remains to prove that there are no edge crossings. By Corollary 1 all edges below the $Z=0$ plane do not cross. We now only consider edges above the $Z=0$ plane.

Each point in an outgoing segment at $v_{a, i}$ has an $X$-coordinate in $[2 a, 2 a+1]$. Thus an outgoing segment at some vertex $v_{a_{1}, i_{1}}$ does not intersect an outgoing segment at some vertex $v_{a_{2}, i_{2}}$ whenever $a_{1} \neq a_{2}$. Clearly an outgoing segment 


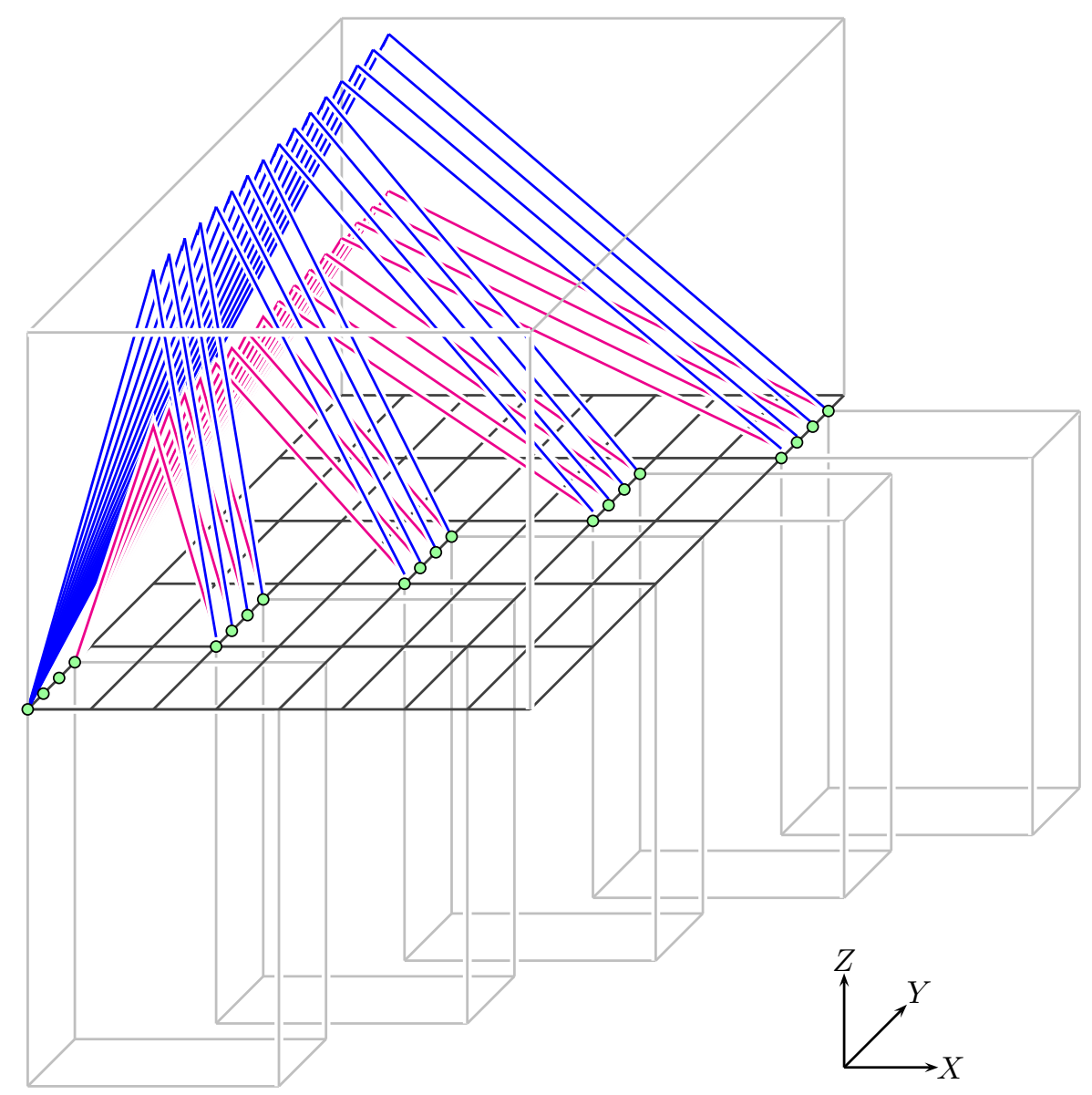

Figure 2: Construction of 1-bend drawing of $K_{n}$ in Theorem 2.

at $v_{a, i_{1}}$ is not coplanar with an outgoing segment at $v_{a, i_{2}}$ whenever $i_{1} \neq i_{2}$, and thus these segments do not cross. Since each bend is assigned a unique gridpoint, any two outgoing segments at the same vertex $v_{a, i}$ do not cross. Thus no two outgoing segments cross.

Each point in an incoming segment at $v_{b, j}$ has a $Y$-coordinate of $b Q+j$. Thus incoming segments at distinct vertices do not cross. Since each bend is assigned a unique gridpoint, any two incoming segments at the same vertex do not cross. Thus no two incoming segments cross.

To prove that an incoming segment does not cross an outgoing segment, we claim that in the projection of the edges on the $Y=0$ plane, an incoming segment does not cross an outgoing segment. In the remainder of the proof we work solely in the $Y=0$ plane, and use $(X, Z)$ coordinates.

The projection in the $Y=0$ plane of an outgoing segment at a vertex $v_{a, i}$ 
is the segment

$$
s_{1}=(2 a, 0) \rightarrow\left(2 a+1,4^{P-a} Q-i\right) .
$$

The projection in the $Y=0$ plane of the incoming segment of an edge $\left(v_{c, k}, v_{d, \ell}\right)$ is the segment

$$
s_{2}=\left(2 c+1,4^{P-c} Q-k\right) \rightarrow(2 d, 0) .
$$

For there to be a crossing clearly we must have $c<a<d$. To prove that there is no crossing it suffices to show that the $Z$-coordinate of $s_{2}$ is greater than the $Z$-coordinate of $s_{1}$ when $X=2 a+1$. Now $s_{2}$ is contained in the line

$$
Z=\frac{4^{P-c} Q-k}{2 c+1-2 d}(X-2 d) \text {. }
$$

Thus the $Z$-coordinate of $s_{2}$ at $X=2 a+1$ is at least

$$
\frac{4^{P-c} Q-Q}{2 c+1-2 d}(2 a+1-2 d)
$$

Thus it suffices to prove that

$$
\frac{4^{P-c} Q-Q}{2 c+1-2 d}(2 a+1-2 d)>4^{P-a} Q
$$

Clearly (1) is implied if it is proved with $a=c+1$ and $d=c+2$. In this case, (1) reduces to

$$
\frac{4^{P-c}-1}{3}>4^{P-c-1}
$$

That is, $4^{P-c-1}>1$, which is true since $c \leq P-2$. This completes the proof.

\section{Acknowledgements}

Thanks to Stephen Wismath for suggesting the problem. 


\section{References}

[1] P. Bose, J. Czyzowicz, P. Morin, and D. R. Wood. The maximum number of edges in a three-dimensional grid-drawing. J. Graph Algorithms Appl., 8(1):21-26, 2004.

[2] T. Calamoneri and A. Sterbini. 3D straight-line grid drawing of 4-colorable graphs. Inform. Process. Lett., 63(2):97-102, 1997.

[3] R. F. Cohen, P. Eades, T. Lin, and F. Ruskey. Three-dimensional graph drawing. Algorithmica, 17(2):199-208, 1996.

[4] O. Devillers, H. Everett, S. Lazard, M. Pentcheva, and S. Wismath. Drawing $K_{n}$ in three dimensions with one bend per edge. In Proc. 13th International Symp. on Graph Drawing (GD '05), Lecture Notes in Comput. Sci. Springer, to appear.

[5] G. Di Battista, P. Eades, R. Tamassia, and I. G. Tollis. Graph Drawing: Algorithms for the Visualization of Graphs. Prentice-Hall, 1999.

[6] E. Di Giacomo, G. Liotta, and H. Meijer. Computing straight-line 3D grid drawings of graphs in linear volume. Comput. Geom., 32(1):26-58, 2005.

[7] J. Díaz, J. Petit, and M. Serna. A survey of graph layout problems. ACM Comput. Surveys, 34(3):313-356, 2002.

[8] R. P. Dilworth. A decomposition theorem for partially ordered sets. Ann. of Math. (2), 51:161-166, 1950.

[9] V. Dujmović, P. Morin, and D. R. Wood. Layout of graphs with bounded tree-width. SIAM J. Comput., 34(3):553-579, 2005.

[10] V. Dujmović and D. R. Wood. On linear layouts of graphs. Discrete Math. Theor. Comput. Sci., 6(2):339-358, 2004.

[11] V. Dujmović and D. R. Wood. Three-dimensional grid drawings with subquadratic volume. In J. Pach, editor, Towards a Theory of Geometric Graphs, volume 342 of Contemporary Mathematics, pages 55-66. Amer. Math. Soc., 2004.

[12] V. Dujmović and D. R. Wood. Stacks, queues and tracks: Layouts of graph subdivisions. Discrete Math. Theor. Comput. Sci., 7:155-202, 2005.

[13] B. Dyck, J. Joevenazzo, E. Nickle, J. Wilsdon, and S. K. Wismath. Drawing $K_{n}$ in three dimensions with two bends per edge. Technical Report TRCS-01-04, Department of Mathematics and Computer Science, University of Lethbridge, 2004.

[14] S. Felsner, G. Liotta, and S. K. Wismath. Straight-line drawings on restricted integer grids in two and three dimensions. J. Graph Algorithms Appl., 7(4):363-398, 2003. 
[15] G. H. Hardy and E. M. Wright. An introduction to the theory of numbers. Clarendon, fifth edition, 1979.

[16] T. Hasunuma. Laying out iterated line digraphs using queues. In G. Liotta, editor, Proc. 11th International Symp. on Graph Drawing (GD '03), volume 2912 of Lecture Notes in Comput. Sci., pages 202-213. Springer, 2004.

[17] M. Kaufmann and D. Wagner, editors. Drawing Graphs: Methods and Models, volume 2025 of Lecture Notes in Comput. Sci. Springer, 2001.

[18] F. T. Leighton and A. L. Rosenberg. Three-dimensional circuit layouts. SIAM J. Comput., 15(3):793-813, 1986.

[19] J. Pach, T. Thiele, and G. Tóth. Three-dimensional grid drawings of graphs. In B. Chazelle, J. E. Goodman, and R. Pollack, editors, Advances in discrete and computational geometry, volume 223 of Contemporary Mathematics, pages 251-255. Amer. Math. Soc., 1999.

[20] C. Ware and G. Franck. Viewing a graph in a virtual reality display is three times as good as a 2D diagram. In A. L. Ambler and T. D. Kimura, editors, Proc. IEEE Symp. Visual Languages (VL '94), pages 182-183. IEEE, 1994.

[21] C. Ware and G. Franck. Evaluating stereo and motion cues for visualizing information nets in three dimensions. ACM Trans. Graphics, 15(2):121140, 1996.

[22] C. Ware, D. Hui, and G. Franck. Visualizing object oriented software in three dimensions. In Proc. IBM Centre for Advanced Studies Conf. (CASCON '93), pages 1-11, 1993. 